\title{
Analysis Of Thin Dielectric Cylindrical Reflector Having An Arbitrary Conic Section Profile Illuminated By Complex Line Source: H-Polarization Case
}

\author{
Taner OGUZER \\ Electrical-Electronics Engineering Dept. \\ Dokuz Eylul University \\ Izmir, TURKIYE \\ taner.oguzer@deu.edu.tr \\ Ibrahim AVGIN \\ Electrical-Electronics Engineering Dept. \\ Ege University \\ Izmir, TURKIYE \\ ibrahim.avgin@ege.edu.tr
}

\author{
Fadil KUYUCUOGLU \\ Electrical-Electronics Engineering Dept. \\ Celal Bayar University \\ Manisa, TURKIYE \\ fadil.kuyucuoglu@cbu.edu.tr \\ Ayhan ALTINTAS \\ Electrical-Electronics Engineering Dept. \\ Bilkent University \\ Ankara, TURKIYE \\ altintas@bilkent.edu.tr
}

\begin{abstract}
Arbitrary conic section profile and thin dielectric reflector is analyzed by using the Method of Analytical Regularization (MAR) technique based on Riemann-Hilbert problem and Fourier inversion procedures. The reflector surface is assumed to be illuminated by an H-polarized complex line source type feed antenna. The convergence of the solution is verified and some changes in the radiation patterns are obtained especially for rather thicker cases. .
\end{abstract}

Index Terms-Dielectric reflector; MAR; scattering

\section{INTRODUCTION}

Curved reflector surfaces have many applications in communication systems. Also they have vast usage areas in the laser technology and optical range systems like the transparent micro devices. Their thicknesses are relatively small compared with the free space wavelength but it may a few fraction of the material wavelength. So there is a phase change between the front and back sides on the reflector. Therefore it is not possible to obtain an accurate data using approximate high frequency techniques like GO, PO and PTD.

Full-wave methods are required for the solution. So, the problem should be formulated by using electromagnetic boundary value problem (BVP) and accurate techniques existing in the literature can solve this BVP. One of the oldest method is finite-difference time domain method. It requires huge number of unknowns due to discretization of large region and far field radiation condition is not forced initially. Another known method is the Method of Moments (MoM). In MoM, the convergence of the solution is not guaranteed and one can expect non-realistic CPU times during simulation in the conventional MoM.
Another accurate, full wave method is the MAR [3]. In MAR, the kernel of the singular integral equations (SIE) is divided into two parts, the more singular part (usually static) and the remainder. The more singular part is analytically inverted by using some special techniques. Finally, it ends up with Fredholm 2nd kind matrix equation with smooth kernel that provides convergent numerical solution. By using the Green's function technique having logarithmic singularity and with some hyper singular parts, the SIE-MAR is extended to 2$\mathrm{D}$ reflectors of noncircular contours. Firstly, their PEC surface cases were analyzed for both polarizations in the literatures. Accurate full wave analysis of the reflector surfaces having imperfect material cases were also studied by SIE-MAR technique. In $[4,5]$, uniform and non-uniform resistive cases of the reflector surface are examined for the noncircular contour for both polarizations.

In the resistive case, very thin layer assumption is valid. But if the thickness is increased more, electric field amplitude in the front side differs from the back side of the layer. Then, the thin layer boundary conditions (TLBC) should be applied as presented in [2]. So, beside electric resistivity (R), magnetic resistivity $(\mathrm{S})$ is required. The cross resistivity $(\mathrm{W})$ is taken as zero. We take transparent layer made of thin dielectric material with some losses. This is a better model of thin layer than resistive counterpart. Thicker layers can be analyzed by this way and it cannot be solved with this much accuracy using resistive model.

The theoretical background of the TLBC based solution by MAR techniques is given in [6] and also various numerical data are given in [7] for flat material strip case. TLBC based solution of the thin dielectric curved reflector surface is analyzed for E-polarization case in [8]. The layer is thin and made up of a high contrast material. This shows the difference 
of the method from low-contrast studies. In this study, the SIEs obtained from TBLC are reduced to two coupled matrix equation systems. One is regularized by Fourier Inversion procedure and other one with the RHP method. The accuracy and convergence are checked by numerical results and some increases are observed in the radiation patterns when layer gets thicker.

\section{FORMULATION}

The problem geometry associated with a thin dielectric with the thickness $h$ and arbitrary profile front fed reflector illuminated by a Complex Source Point (CSP) feed is presented in Figure 1. The 2-D cross section of the reflector is defined as conic-section profile and it may be an elliptic, parabolic or hyperbolic arc. Here we only note that they differ in the value of so-called eccentricity, e, so that $\mathrm{e}<1$ for an ellipse; $\mathrm{e}=1$ for a parabola and e $>1$ for a hyperbola.

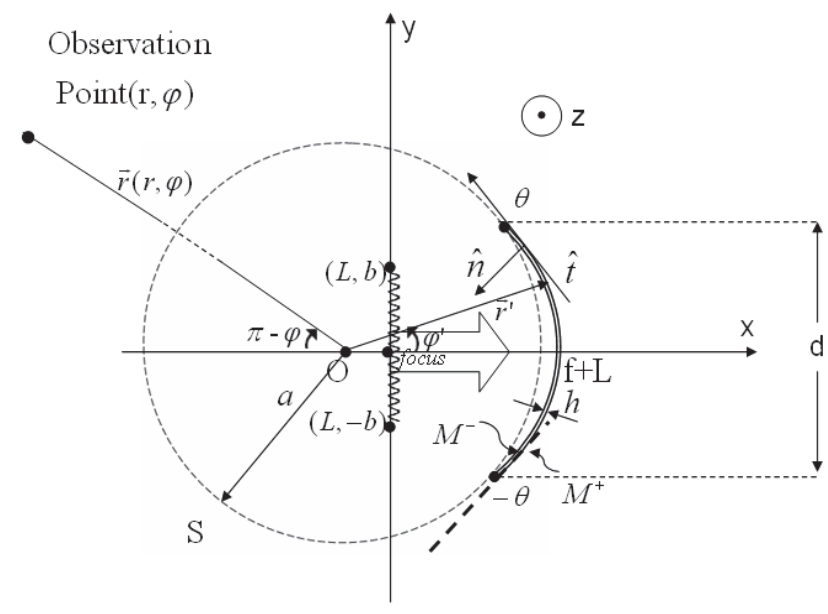

Figure 1. Problem Geometry

The feed is located at the near geometrical focus of the reflector and a closed contour is defined as $\mathrm{C}$ that is the completed the reflector surface $\mathrm{M}$ by a aperture $\mathrm{S}$. Additionally one thing has to be said that the M(metal) part of the reflector surface does not cross the CSP branch and also the circular arc $\mathrm{S}$ of the total contour $\mathrm{C}$ must be continuous curvature at the reflector edge points.

The rigorous formulation of the considered boundary value problem can be stated in terms of the Helmholtz equation, the Sommerfeld radiation condition far from the reflector and source, the thin layer boundary conditions on $\mathrm{M}$, and an edge condition such that the field energy is limited in any finite domain around the reflector edge. Collectively, these conditions guarantee the uniqueness of the problem solution [1].

The thin layer boundary conditions are a wellestablished model of a thin penetrable material sheet. It can be written as the following equations:

$\left[\vec{E}_{T}^{+}(\vec{r})+\vec{E}_{T}^{-}(\vec{r})\right]=2 R(\vec{r}) \underbrace{\hat{n}(\vec{r}) \times\left[\vec{H}_{T}^{+}(\vec{r})-\vec{H}_{T}^{-}(\vec{r})\right]}_{\vec{J}_{z}}, \quad \vec{r} \in M$
$\left[\vec{H}_{T}^{+}(\vec{r})+\vec{H}_{T}^{-}(\vec{r})\right]=2 S(\vec{r}) \underbrace{\hat{n}(\vec{r}) \times\left[\vec{E}_{T}^{-}(\vec{r})-\vec{E}_{T}^{+}(\vec{r})\right]}_{\vec{M}_{t}}, \quad \vec{r} \in M$

where subscript " $T$ " indicates the tangential field, the superscripts ". " and "+" relate to the front and back faces of reflector, respectively, and the unit normal vector $\hat{n}$ is directed to the inside that the feed is located. The unknown surface current densities $\mathrm{J}_{\mathrm{t}}$ and $\mathrm{M}_{\mathrm{z}}$ are obtained for H-polarization case where $\mathrm{H}$ field polarized along $\mathrm{z}$-direction.

For relatively thin layer $\left(\mathrm{k}_{\mathrm{o}} \mathrm{h}<<1\right), \mathrm{R}_{\mathrm{T}}$ and $\mathrm{S}_{\mathrm{T}}$ parameters can be written as follows,

$R_{T}=\frac{i}{2} \sqrt{\frac{\varepsilon}{\mu}} \cot \left(\frac{1}{2} \sqrt{\varepsilon \mu /\left(\varepsilon_{0} \mu_{0}\right)} k_{0} h\right) \quad S_{T}=\frac{i}{2} \sqrt{\frac{\mu}{\varepsilon}} \cot \left(\frac{1}{2} \sqrt{\varepsilon \mu /\left(\varepsilon_{0} \mu_{0}\right)} k_{0} h\right)$

These $\mathrm{R}_{\mathrm{T}}$ and $\mathrm{S}_{\mathrm{T}}$ parameters are defined for infinite flat sheets and also $\left|\varepsilon_{r}\right| \gg>1$ assumption is considered (that is the high contrast case). These can also be used for the smoothly curved surface.

The incident magnetic field and the tangential electric field will be taken as the beam-like form generated by the CSP, given as

$$
H_{z}^{i n}(\vec{r})=H_{0}^{(1)}\left(k\left|\vec{r}-\vec{r}_{s}\right|\right) \quad E_{T}^{i n}(\vec{r})=-\frac{1}{i \omega \varepsilon} \frac{\partial H_{z}^{i n c}(\vec{r})}{\partial n}
$$

where $\vec{r}_{s}=\left(x_{0}+i b \cos \beta, y_{0}+i b \sin \beta\right)$. This incident field function has two branch points which should be connected with a branch cut. Its maximum is in the along $\varphi=\beta$ direction. Also parameters $b$ and $\beta$ are the aperture width and beam aiming angle respectively. Also $\left(\mathrm{x}_{0}, \mathrm{y}_{0}\right)$ indicates the real position coordinates.

With the aid of the auxiliary potential expressions and by using TLBC, the following SIE's can be obtained as,

$$
\begin{aligned}
& j \omega \varepsilon \int_{M} M_{Z}\left(\vec{r}^{\prime}\right) G\left(k_{o}\left|\vec{r}-\vec{r}^{\prime}\right|\right) d l^{\prime} \\
& +\int_{M} J_{t}\left(\vec{r}^{\prime}\right) \frac{\partial}{\partial n^{\prime}} G\left(k_{o}\left|\vec{r}-\vec{r}^{\prime}\right|\right) d l^{\prime} \\
& +H_{z}^{i n}=S_{T} M_{z},(\vec{r} \in M) \text { and } M_{z}=0(\vec{r} \in S) \\
& \frac{i}{\omega \varepsilon} \int_{M} J_{t}\left(\vec{r}^{\prime}\right) \frac{\partial}{\partial n \partial n^{\prime}} G\left(k_{o}\left|\vec{r}-\vec{r}^{\prime}\right|\right) d l^{\prime} \\
& -\int_{M} M_{Z}\left(\vec{r}^{\prime}\right) \frac{\partial}{\partial n} G\left(k_{o}\left|\vec{r}-\vec{r}^{\prime}\right|\right) d l^{\prime}+E_{T}^{i n} \\
& =R_{T} J_{t},(\vec{r} \in M) \text { and } J_{t}=0(\vec{r} \in S)
\end{aligned}
$$

where the scalar Green's function G is a Hankel function of the zero order and first kind satisfying the radiation condition; i.e. $\quad G\left(\vec{r}(\varphi), \vec{r}^{\prime}\left(\varphi^{\prime}\right)\right)=(i / 4) H_{0}^{(1)}\left(k_{o} R\right)$, where $R=\left|\vec{r}(\varphi)-\vec{r}^{\prime}\left(\varphi^{\prime}\right)\right|$. Assume that the curve $\mathrm{M}$ can be characterized with the aid of the parametric equations $x=x(\varphi), y=y(\varphi) \quad 0 \leq|\varphi| \leq \theta$ in terms of polar angle, $\varphi$. Besides define the differential lengths in the tangential direction at any point on $\mathrm{M}$ as $\partial l=a \beta(\varphi) \partial \varphi$, $\partial l^{\prime}=a \beta\left(\varphi^{\prime}\right) \partial \varphi^{\prime} \quad$ respectively.

Also 
$\beta(\varphi)=r(\varphi) /(a \cos \gamma(\varphi)), \xi(\varphi)$ is the angle between the normal on $\mathrm{M}$ and the $\mathrm{x}$-direction, $\gamma(\varphi)$ is the angle between the normal and the radial direction. Then we extend the surface-current densities $M_{z}$ and $J_{t}$ with zero value to $\mathrm{S}$. Then the above sets of equations are modified on the complete contour C (MUS).

The simple parameter change type duality in the resultant solution does not work. For the H polarization type coupled dual SIE systems compared with the E-polarization case, some sign changes and $\varepsilon$ replacement by $\mu$ produces the different radiation performance.

To proceed with the MAR based formulation all functions should be expressed in terms of the Fourier series form. Firstly the incident fields are explained in terms of the FS coefficients. Furthermore to work with this formulation and make computations more economic, we add and subtract the similar functions from the originally givens. These functions are defined at the full circle of the closed contour $\mathrm{C}$ and they are introduced in [9].

Some of these functions have also continuous first derivatives and their second derivatives with respect to $\varphi$ and $\varphi$ ' have only logarithmic singularity and hence belong to $L_{2}$. Therefore their Fourier Series (FS) coefficients decay as $O\left(|n|^{-1.5-\varepsilon}|m|^{-1.5-\varepsilon}\right)$ on C. Some other two functions have no singularity at all even in their each term. So it is expected that they decay faster. The corresponding FS coefficients of these functions can be computed by FFT algorithm effectively. So this provides us to solve reasonably larger geometries.

Firstly the SIE type dual system in equation (5) is solved by Fourier inversion procedure. The other system in equation (6) is converted to a dual series form and then it is regularized by RHP technique. Finally we obtained two coupled algebraic equation system and they build a matrix system as follows.

$$
\left(\left[\begin{array}{ll}
I & 0 \\
0 & I
\end{array}\right]-\left[\begin{array}{ll}
A_{m n} & B_{m n} \\
D_{m n} & C_{m n}
\end{array}\right]\right)\left[\begin{array}{l}
x_{n} \\
m_{n}
\end{array}\right]=\left[\begin{array}{c}
T_{m} \\
E_{m}
\end{array}\right]
$$

where $x_{m}$ and $m_{m}$ are the FS coefficients for $M_{z}$ and $J_{t}$ surface current density functions respectively. Then together with the large asymptotic expansions for the cylindrical functions, one can find that $\sum_{m, n=-\infty}^{\infty}\left|Z_{m n}\right|^{2}<\infty$. By using the similar procedure, one can find that $\sum_{m=-\infty}^{\infty}\left|P_{m}\right|^{2}<\infty$ provided that the branch cut associated with the CSP aperture does not cross the reflector contour M. Finally obtained the infinite matrix equation (7) is of the Fredholm $2^{\text {nd }}$ kind. Hence the Fredholm theorems guarantee the existence of the unique exact solution and also the convergence of approximate numerical solution when truncating (7) with progressively larger sizes $\mathrm{N}_{\mathrm{tr}}$.

\section{NUMERICAL RESULTS}

Above presented formulation is tested by written computer codes to obtain unknown current density functions, radiation patterns and directivities. Figure 2(a) presents the forward directivity obtained from MAR versus Ntr for three different eccentricity factors. As expected, while the truncation number increasing, the all directivities converge and the parabolic surface has the highest one. In Figure 2(b), the relative accuracy in directivity defined as $\Delta \mathrm{D}=\left|\mathrm{D}^{\mathrm{N}_{\mathrm{tr}}+1}-\mathrm{D}^{\mathrm{N}_{\mathrm{tr}}}\right| / \mathrm{D}^{\mathrm{N}_{\mathrm{tr}}}$ is given in logarithmic scale. These errors also decay smoothly and so the convergence nature of the given formulation is examined.
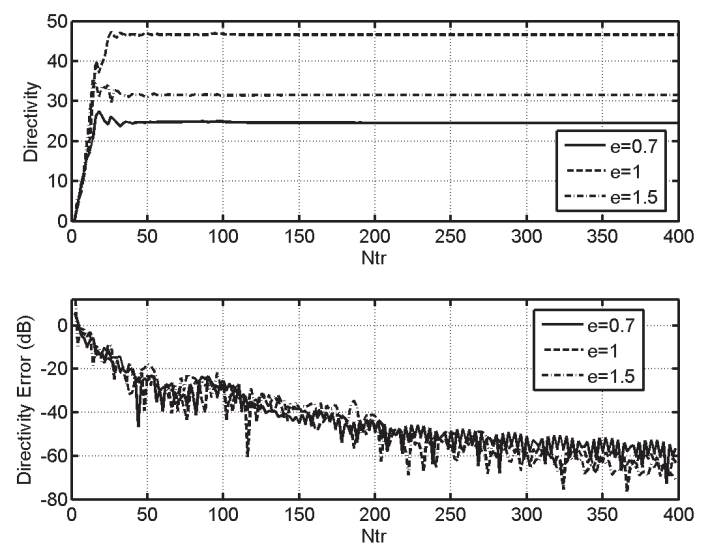

Figure 2: (a) Directivity versus Ntr (b) The relative error in directivity The other parameters are $\mathrm{f}=10 \lambda, \mathrm{d}=15 \lambda, \mathrm{kb}=5, \mathrm{~h}=0.2 \lambda_{\mathrm{e}}, \in_{\mathrm{r}}=20+2 \mathrm{i}, \mu_{\mathrm{r}}=1$

Figure 3 shows surface currents and the radiation patterns for different thicknesses. Increase in the radiated field level is observed at the penumbra region between $20^{\circ}$ and $120^{\circ}$, as seen in Figure 3(c). This situation is seen to be higher for thicker layers and observed for only for H-polarization case. In the region where the field level is increasing, surface current densities are becoming more oscillatory. (See Figure 3(a) and 3(b)). This implies that the interfering fields inside the layer become more dominant for thicker layers and so stronger standing waves can occur. The increase in the radiation patterns can be attributed to these oscillatory currents. 

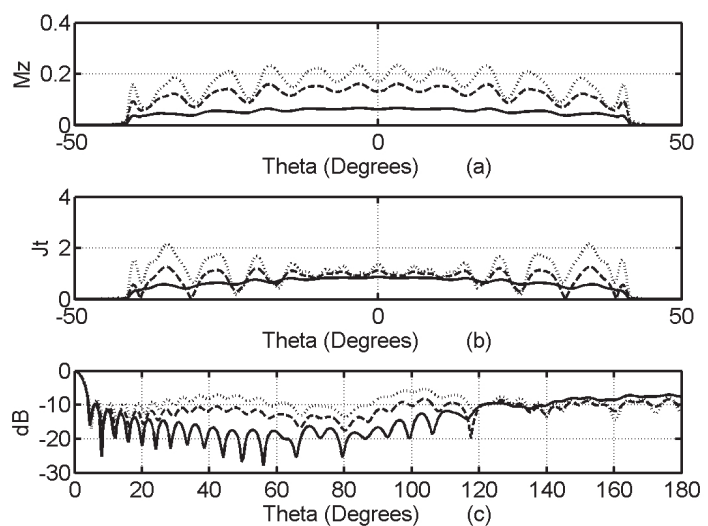

Figure 3. $\mathrm{f}=10 \lambda, \mathrm{d}=15 \lambda, \mathrm{e}=1, \mathrm{~kb}=3, \epsilon_{\mathrm{r}}=1$, (a) The current density $\mathrm{Mz}$ (b) The current density $\mathrm{Jt} \quad$ (c) The normalized radiation pattern

The solidline $\mathrm{h}=0.1 \lambda_{\mathrm{e}}$, dashedline $\mathrm{h}=0.2 \lambda_{\mathrm{e}}$, dotted line $\mathrm{h}=0.25 \lambda_{\mathrm{e}}$.

\section{CONCLUSION}

The arbitrary conic section profile and penetrable low loss reflector is illuminated by H-polarized CSP. The problem is formulated as the electromagnetic BVP. It was analyzed and modeled by a combined semi-inversion approach for $\mathrm{H}$ polarization. Simulation results were shown that the presented formulation really converges and great compatibility observed with resistive case for thin layers. The radiation patterns imply that some increase is observed at the penumbra region.

\section{REFERENCES}

[1] D. Colton and R. Kress, Integral Equation Method in Scattering Theory, New York, Wiley, 1983.

[2] E. Bleszynski, M. Bleszynski, and T. Jaroszewicz, "Surface-integral equations for electromagnetic scattering from impenetrable and penetrable sheets," IEEE Anten. Propag. Mag., vol. 35, no 6, pp. 14-25, 1993.

[3] A.I. Nosich, "The method of analytical regularization in wave-scattering and eigenvalue problems: foundations and review of solutions," IEEE Trans. Anten. Propag. Mag., vol. 41, no 3, pp. 34-49, 1999.

[4] Oğuzer T, Altintas A, Nosich A. I., "Integral equation analysis of an arbitrary profile and varying-resistivity cylindrical reflector illuminated by an E-polarized complex source-point beam." J. Opt. Soc. Am. A, vol. 26, no 7, pp. $1525-1532,2009$

[5] Oğuzer T, Altintas A, Nosich A. I., "Analysis of the elliptic-profile cylindrical reflector with a varying resistivity using the complex source and dual-series approach: H-polarization case," Opt. Quantum Electron, vol. 45, no 8, pp. 797-812, 2013.

[6] Alexander I. Nosich, "Method of analytical regularization based on the static part inversion in wave scattering by imperfect thin screens," J. Telecomm. and Inf. Tech., Warsaw, NIT Press, no 3, pp. 34-49, 1999.

[7] Olga V. Shapoval, Ronan Sauleau and Alexander I. Nosich, "Scattering and absorption of waves by flat material strips analyzed using generalized boundary conditions and Nystrom-type algorithm" IEEE Trans. Anten. and Propag., vol. 59, no 9, 2011.

[8] Taner Oğuzer, Fadıl Kuyucuoğlu, Ibrahim Avgin and Ayhan Altintas, "Analysis of an arbitrary conic section profile and thin dielectric cylindrical reflector illuminated by an E-polarized complex source point beam," Proc. Int. Mathem. Methods in Electromagnetic Theory (MMET12), Kharkiv, pp. 292295.

[9] Fadil Kuyucuoglu, Taner Oğuzer, Ibrahim Avgin and Ayhan Altintas, "Analysis of an arbitrary-profile, cylindrical, impedance reflector surface illuminated by an E-polarized complex line source beam," Journal of Electromagnetic Waves and Applications, vol. 28, no 3, pp. 360-377, 2013. 